Wilhelm Rudorf, Müncheberg, Mark: Natürliche Auslese.

Wilhelm Rudorf, Müncheberg, Mark: Entwicklungsphysiologische Grundlagen der Pflanzenzüchtung.

ThEodor RoEMer, Halle a. S.: Ertragssicherheit - Krankheitsresistenz.

Walter H. Fuchs, Halle a. S. und Klaus v. Rosenstiel, Müncheberg, Mark: Physiologische Resistenz.

KARL Wetzel, Berlin: Die physiologischen Grundlagen der pflanzlichen Stoffproduktion.

Olaf Tedin, Svalöf: Biologische Statistik.

'THeodor Roemer, Halle a. S.: Methoden und Technik der Auslese.

Theodor Roemer, Halle a. S.: Úberblick über die Methoden der Züchtung.

Theodor Roemer, Halle a. S.: Auslesezüchtung.

Wilhelm Rudorf, Müncheberg, Mark: Kreuzung innerhalb der Art.

ERnst Oehler, Müncheberg, Mark: Art- und Gattungsbastarde.

Ëdgar Knapp, Müncheberg, Mark: Züchtung durch Mutationslösung.

Karl Isenbech, Halle a. S.: Die Prüfung des Zuchterfolges.

Mit diesem Werk ist die für den Einzelnen unübersehbare Fülle von Forschungsergebnissen auf den Gebieten der landwirtschaftlichen und gartenbaulichen Züchtung, der Botanik, Vererbungslehre, Physiologie, Biochemie und anderer Grenzgebiete gesichtet und verarbeitet, was für den weiteren Fortschritt auf allen Gebieten der Züchtung von unschätzbarem Vorteil ist. Das neue Handbuch gibt jedem wissenschaftlich oder praktisch arbeitenden Pflanzenzüchter das Rüstzeug gesicherter Erkenntnisse in die Hand, um auf dem beschrittenen Weg erfolgreich weiterzuarbeiten. Damit steht in Aussicht, daf die landwirtschaftliche Erzeugung weiterhin gesteigert und unserem Volk die Ernährungsfreiheit gesichert werden wird. Die meisterhafte, kaum zu übertreffende Darstellung der Grundlagen der Pflanzenzüchtung im I. Band des Handbuches wird auch für die zwar immer noch in den Anfängen steckende, aber im Hinblick auf die Holzknappheit und den künftigen gesteigerten Holzbedarf unerläßliche und mit allem Nachdruck aufzubauende forstliche Züchtung befruchtend und nutzbringend verwertet werden können.

ROHMEDER

\title{
IV. KURZE NACHRICHTEN
}

\section{Studienreise der Internationalen Forstzentrale nach Hessen}

Vom 18.-22. Mai unternahmen die ständigen Mitarbeiter der Internationalen Forstzentrale unter Leitung des Direktors Prof. Dr. Dr. Köstlek eine Studienreise nach Hessen.

Landesforstmeister Dr. h. c. Hesse und eine größere Zahl von Staats- und Privatforstbeamten leiteten die Exkursion durch die Waldgebiete des Vogelbergs, des Odenwalds und der Rheinebene. In Vorträgen und Aussprachen wurden bei den Wallbesichtigungen besonders die Fragen der Buchenbewirtschaftung, der Behandlung zersplitterten Privatwaldbesitzes und des Eichenschälwaldes behandelt. 'In diesen und anderen Fragen wurden die durch die Kriegswirtschaft gestellten Aufgaben erörtert. Die Reise gestaltete sich in jeder Richtung als äußerst wertvoll für die Arbeitsaufgaben der Internationalen Forstzentrale. 